\title{
Self-adaptive moving mesh schemes for short pulse type equations and their Lax pairs
}

\author{
Bao-Feng Feng ${ }^{1}$, Kenichi Maruno ${ }^{1,2^{*}}$ and Yasuhiro Ohta ${ }^{3}$
}

\begin{abstract}
Integrable self-adaptive moving mesh schemes for short pulse type equations (the short pulse equation, the coupled short pulse equation, and the complex short pulse equation) are investigated. Two systematic methods, one is based on bilinear equations and another is based on Lax pairs, are shown. Self-adaptive moving mesh schemes consist of two semi-discrete equations in which the time is continuous and the space is discrete. In self-adaptive moving mesh schemes, one of two equations is an evolution equation of mesh intervals which is deeply related to a discrete analogue of a reciprocal (hodograph) transformation. An evolution equations of mesh intervals is a discrete analogue of a conservation law of an original equation, and a set of mesh intervals corresponds to a conserved density which play an important role in generation of adaptive moving mesh. Lax pairs of self-adaptive moving mesh schemes for short pulse type equations are obtained by discretization of Lax pairs of short pulse type equations, thus the existence of Lax pairs guarantees the integrability of self-adaptive moving mesh schemes for short pulse type equations. It is also shown that self-adaptive moving mesh schemes for short pulse type equations provide good numerical results by using standard time-marching methods such as the improved Euler's method.
\end{abstract}

Keywords: Solitons; Self-adaptive moving mesh schemes; Short pulse equation

\section{Introduction}

The studies of discrete integrable systems were initiated in the middle of 1970 s. Hirota discretized various soliton equations such as the $\mathrm{KdV}$, the $\mathrm{mKdV}$, and the sine-Gordon equations based on the bilinear equations [16-20], Ablowitz and Ladik proposed a method of integrable discretizations of soliton equations, including the nonlinear Schrödinger equation and the modified $\mathrm{KdV}$ $(\mathrm{mKdV})$ equation, based on the Ablowitz-Kaup-NewellSegur (AKNS) form [1-5]. Following the pioneering works of Hirota and Ablowitz-Ladik, the studies of discrete integrable systems have been expanded in diverse areas (see, for example, $[6,7,15,40])$.

It is known that there is a class of soliton equations which are derived from the Wadati-Konno-Ichikawa (WKI) type $2 \times 2$ linear system $[5,27,43]$. Soliton equations in the WKI class are transformed to certain soliton

*Correspondence: kmaruno@waseda.jp

1 Department of Mathematics, The University of Texas-Pan American, Edinburg, Texas 78541, USA

2 Department of Applied Mathematics, School of Fundamental Science and Engineering, Waseda University, 3-4-1 Okubo, Shinjuku-ku, Tokyo 169-8555, Japan

Full list of author information is available at the end of the article equations which are derived from the AKNS type $2 \times 2$ linear system through reciprocal (hodograph) transformations $[5,22,23,36,44]$.

Integrable discretization of soliton equations in the WKI class had been regarded as a difficult problem until recently. A systematic treatment of reciprocal (hodograph) transformations in integrable discretizations had been unknown for three decades. Recently, the present authors proposed integrable discretizations of some soliton equations in the WKI class by using the bilinear method, and it was confirmed that those integrable discrete equations work effectively on numerical computations of the above class of soliton equations as selfadaptive moving mesh schemes [11-14,34,35]. However, the method employed in our previous papers was rather technical, thus it is not easy to extract a fundamental structure of discretizations to apply this method to a broader class of nonlinear wave equations including nonintegrable systems.

The aim of this article is to present two systematic methods (in sophisticated forms), one is based on bilinear equations (this method is regarded as an extension of Hirota's discretization method) and another is based

\section{Springer}

(c) 2014 Feng et al.; licensee Springer. This is an Open Access article distributed under the terms of the Creative Commons Attribution License (http://creativecommons.org/licenses/by/4.0), which permits unrestricted use, distribution, and reproduction in any medium, provided the original work is properly credited. 
on Lax pairs (this method is regarded as an extension of Ablowitz-Ladik's discretization method), to construct self-adaptive moving mesh schemes for soliton equations in the WKI class. We demonstrate how to construct self-adaptive moving mesh schemes for short pulse type equations whose Lax pairs are written in the WKI type form which is transformed into the Ablowitz-KaupNewell-Segur (AKNS) type form by reciprocal (hodograph) transformations. We clarify that moving mesh is generated by following discrete conservation law and mesh intervals are nothing but discrete conserved densities which is a key of self-adaptive moving mesh schemes. Lax pairs of self-adaptive moving mesh schemes for short pulse type equations are constructed by discretization of Lax pairs of short pulse type equations. It is also shown that self-adaptive moving mesh schemes for short pulse type equations provide good numerical results by using standard time-marching methods such as the improved Euler's method.

The short pulse (SP) equation [8,32,33,37-39]

$$
u_{x t}=u+\frac{1}{6}\left(u^{3}\right)_{x x},
$$

is linked with the so-called coupled dispersionless (CD) system $[21,26,28,29]$

$$
\begin{aligned}
& \frac{\partial \rho}{\partial T}-\frac{\partial}{\partial X}\left(-\frac{u^{2}}{2}\right)=0, \\
& \frac{\partial^{2} u}{\partial X \partial T}=\rho u,
\end{aligned}
$$

through the reciprocal transformation (this is often called the hodograph transformation in many literatures)

$$
x=X_{0}+\int_{X_{0}}^{X} \rho(\tilde{X}, T) d \tilde{X}, \quad t=T,
$$

where $X_{0}$ is a constant. The reciprocal (hodograph) transformation (4) yields

$$
\begin{aligned}
& \frac{\partial}{\partial X}=\rho \frac{\partial}{\partial x}, \\
& \frac{\partial}{\partial T}=\frac{\partial}{\partial t}-\frac{u^{2}}{2} \frac{\partial}{\partial x} .
\end{aligned}
$$

Note that the reciprocal (hodograph) transformation (4) originates from the conservation law (2). Applying the reciprocal (hodograph) transformation (4) to (3) yields

$$
\frac{\partial}{\partial x}\left(\frac{\partial}{\partial t}-\frac{u^{2}}{2} \frac{\partial}{\partial x}\right) u=u \text {. }
$$

This can be rewritten as eq. (1). Thus the SP equation is equivalent to the $\mathrm{CD}$ system with the reciprocal (hodograph) transformation. As we mentioned in our previous paper, the reciprocal (hodograph) transformation between the CD system and the SP equation is nothing but the transformation between the Lagrangian coordinate and the Eulerian coordinate [11].

The CD system (2) and (3) can be derived from the compatibility condition of the following linear $2 \times 2$ system (Lax pair) [28]:

$$
\frac{\partial \Psi}{\partial X}=U \Psi, \quad \frac{\partial \Psi}{\partial T}=V \Psi
$$

where

$$
U=-\mathrm{i} \lambda\left(\begin{array}{cc}
\rho & u_{X} \\
u_{X} & -\rho
\end{array}\right), \quad V=\left(\begin{array}{cc}
\frac{\mathrm{i}}{4 \lambda} & -\frac{u}{2} \\
\frac{u}{2} & -\frac{1}{4 \lambda}
\end{array}\right),
$$

and $\Psi$ is a two-component vector. By applying the reciprocal (hodograph) transformation (4) into the above linear $2 \times 2$ system (Lax pair) (8) and (9), we obtain the linear $2 \times 2$ system (Lax pair) for the short pulse equation [38]:

$$
\frac{\partial \Psi}{\partial x}=\tilde{U} \Psi, \quad \frac{\partial \Psi}{\partial t}=\tilde{V} \Psi
$$

where

$$
\begin{aligned}
& \tilde{U}=-\mathrm{i} \lambda\left(\begin{array}{cc}
1 & u_{x} \\
u_{x} & -1
\end{array}\right), \\
& \tilde{V}=\left(\begin{array}{cc}
\frac{\mathrm{i}}{4 \lambda}-\frac{\mathrm{i} \lambda}{2} u^{2} & -\frac{u}{2}-\frac{\mathrm{i} \lambda}{2} u^{2} u_{x} \\
\frac{u}{2}-\frac{\mathrm{i} \lambda}{2} u^{2} u_{x} & -\frac{\mathrm{i}}{4 \lambda}+\frac{\mathrm{i} \lambda}{2} u^{2}
\end{array}\right),
\end{aligned}
$$

which can be rewritten as

$$
\begin{aligned}
& \tilde{U}=\lambda\left(\begin{array}{cc}
1 & u_{x} \\
u_{x} & -1
\end{array}\right), \\
& \tilde{V}=\left(\begin{array}{cc}
\frac{1}{4 \lambda}+\frac{\lambda}{2} u^{2} & -\frac{u}{2}+\frac{\lambda}{2} u^{2} u_{x} \\
\frac{u}{2}+\frac{\lambda}{2} u^{2} u_{x} & -\frac{1}{4 \lambda}-\frac{\lambda}{2} u^{2}
\end{array}\right),
\end{aligned}
$$

by replacing $\lambda$ by $i \lambda$. This is nothing but the Lax pair of the SP equation. Note that the Lax pair of the SP equation is of the WKI type [38]. In general, soliton equations derived from WKI-type eigenvalue problems are transformed into soliton equations derived from AKNS-type eigenvalue problems by reciprocal (hodograph) transformations $[22,23,36,44]$.

\section{A self-adaptive moving mesh scheme for the SP equation and its Lax pair}

The SP equation (1) can be discretized by means of the following two methods:

\section{Method 1: The discretization method using bilinear equations}

- Step 1: Transform the SP equation (1) into the CD system (2) and (3) by the reciprocal (hodograph) transformation (4).

- Step 2: Transform the CD system into the bilinear equations.

- Step 3: Discretize the bilinear equations of the CD system.

- Step 4: Transform the (semi-)discrete bilinear equations into the (semi-)discrete CD system. 
- Step 5: Discretize the reciprocal (hodograph) transformation and transform the (semi-)discrete CD system via the discrete reciprocal (hodograph) transformation.

\section{Method 2: The discretization method using a Lax pair}

- Step 1: Transform the Lax pair of the SP equation (1) by the reciprocal (hodograph) transformation (4). The Lax pair obtained by the reciprocal (hodograph) transformation is the one of the CD system (2) and (3).

- Step 2: Discretize the Lax pair of the CD system. The compatibility condition of the discretized Lax pair yields the (semi-)discrete CD system.

- Step 3: Discretize the reciprocal (hodograph) transformation and transform the discretized Lax pair of the (semi-)discrete CD system via the discrete reciprocal (hodograph) transformation.

- Step 4: The compatibility condition of the discretized Lax pair obtained in Step 3 yields the (semi-)discrete SP equation.

Since the SP equation (1) is equivalent to the CD system (2) and (3) with the reciprocal (hodograph) transformation (4), the semi-discrete CD system with the discrete reciprocal (hodograph) transformation is equivalent to the semi-discrete SP equation.

Here we show the details of procedures to construct the self-adaptive moving mesh scheme for the SP equation by means of the above two methods.

\section{Method 1:}

Step 1: The SP equation (1) is transformed into the CD system (2) and (3) via the reciprocal (hodograph) transformation (4).

Step 2: The CD system (2) and (3) can be transformed into the bilinear equations

$$
\begin{aligned}
& D_{T}^{2} f \cdot f=\frac{1}{2} g^{2}, \\
& D_{X} D_{T} f \cdot g=f g,
\end{aligned}
$$

via the dependent variable transformation

$$
u=\frac{g}{f}, \quad \rho=1-2(\ln f)_{X T} .
$$

Here $D_{X}$ and $D_{T}$ are Hirota's $D$-operators defined as

$$
D_{X}^{m} f \cdot g=\left.\left(\partial_{X}-\partial_{X^{\prime}}\right)^{m} f(X) g\left(X^{\prime}\right)\right|_{X^{\prime}=X} .
$$

Step 3: Discretize the space variable $X$ in the bilinear equations (15) and (16).

$$
D_{T}^{2} f_{k} \cdot f_{k}=\frac{1}{2} g_{k}^{2}
$$

$$
\begin{aligned}
\frac{1}{a} D_{T}\left(f_{k+1} \cdot g_{k}-f_{k} \cdot g_{k+1}\right) \\
=\frac{1}{2}\left(f_{k+1} g_{k}+f_{k} g_{k+1}\right) .
\end{aligned}
$$

Step 4: Consider the dependent variable transformation

$$
u_{k}=\frac{g_{k}}{f_{k}}, \quad \rho_{k}=1-\frac{2}{a}\left(\ln \frac{f_{k+1}}{f_{k}}\right)_{T},
$$

which is a discrete analogue of (17). Then the bilinear equations (18) and (19) are transformed into

$$
\begin{aligned}
& \partial_{T} \rho_{k}-\frac{\left(-\frac{u_{k+1}^{2}}{2}\right)-\left(-\frac{u_{k}^{2}}{2}\right)}{a}=0, \\
& \partial_{T}\left(\frac{u_{k+1}-u_{k}}{a}\right)=\rho_{k} \frac{u_{k+1}+u_{k}}{2},
\end{aligned}
$$

which is a semi-discrete analogue of the $C D$ system.

Step 5: Consider a discrete analogue of the reciprocal (hodograph) transformation

$$
x_{k}=X_{0}+\sum_{j=0}^{k-1} a \rho_{j},
$$

where $x_{0}=X_{0}$. Now we introduce the mesh interval

$$
\delta_{k}=x_{k+1}-x_{k} .
$$

Note that the mesh interval satisfies the relation

$$
\delta_{k}=a \rho_{k},
$$

so we can rewrite equations (21) and (22) with the discrete reciprocal (hodograph) transformation (23) into the self-adaptive moving mesh scheme for the SP equation

$$
\begin{aligned}
& \partial_{T} \delta_{k}=\frac{-u_{k+1}^{2}+u_{k}^{2}}{2}, \\
& \partial_{T}\left(u_{k+1}-u_{k}\right)=\delta_{k} \frac{u_{k+1}+u_{k}}{2},
\end{aligned}
$$

where $\delta_{k}$ is related to $x_{k}$ by $\delta_{k}=x_{k+1}-x_{k}$ which originates from the discrete reciprocal (hodograph) transformation

$$
x_{k}=X_{0}+\sum_{j=0}^{k-1} \delta_{j} .
$$

The set of points $\left\{\left(x_{k}, u_{k}\right)\right\}_{k=0,1, \cdots}$ provides a solution of the semi-discrete SP equation. Note that the above discrete reciprocal (hodograph) transformation can be interpreted as the transformation between Eulerian description and Lagrangian description in a discretized space [11]. 
The discrete reciprocal (hodograph) transformation (28) yields

$$
\begin{aligned}
\frac{\Delta}{\Delta X_{k}} & =\frac{\Delta}{a}=\frac{\Delta x_{k}}{a} \frac{\Delta}{\Delta x_{k}}=\rho_{k} \frac{\Delta}{\Delta x_{k}}=\rho_{k} \frac{\Delta}{\delta_{k}} \\
\frac{\partial}{\partial T} & =\frac{\partial}{\partial t}+\frac{\partial x_{k}}{\partial T} \frac{\partial}{\partial x_{k}}=\frac{\partial}{\partial t}+\sum_{j=0}^{k-1} \frac{\partial \delta_{j}}{\partial T} \frac{\partial}{\partial x_{k}} \\
& =\frac{\partial}{\partial t}+\left(\sum_{j=0}^{k-1} \frac{-u_{j+1}^{2}+u_{j}^{2}}{2}\right) \frac{\partial}{\partial x_{k}} \\
& =\frac{\partial}{\partial t}+\left(\frac{-u_{k+1}^{2}+u_{0}^{2}}{2}\right) \frac{\partial}{\partial x_{k}} \\
& =\frac{\partial}{\partial t}+\left(\frac{-u_{k+1}^{2}}{2}\right) \frac{\partial}{\partial x_{k}}, \quad \text { if } u_{0}=0
\end{aligned}
$$

where $\Delta$ is a difference operator defined as $\Delta f_{k} \equiv f_{k+1}-f_{k}$. Applying this to eq. (27), we obtain

$$
\begin{gathered}
\frac{1}{\delta_{k}} \frac{\partial\left(u_{k+1}-u_{k}\right)}{\partial t}-\frac{u_{k+1}^{2}}{2} \frac{1}{\delta_{k}} \frac{\partial\left(u_{k+1}-u_{k}\right)}{\partial x_{k}} \\
=\frac{u_{k+1}+u_{k}}{2} .
\end{gathered}
$$

In the continuous limit $\delta_{k} \rightarrow 0$, this leads to the SP equation (1).

We remark that eq. (21) describes the evolution of the mesh interval $\delta_{k}$, and this equation is nothing but a discrete analogue of the conservation law (2). This means that the mesh interval $\delta_{k}$ is a conserved density of the self-adaptive moving mesh scheme. Thus the mesh interval $\delta_{k}$ is determined by the semi-discrete conservation law. From the semi-discrete conservation law, one can find the following property: If $\frac{-u_{k+1}^{2}+u_{k}^{2}}{2}<0$, i.e., the slope between $u_{k}^{2}$ and $u_{k+1}^{2}$ is positive, then the mesh interval $\delta_{k}$ becomes smaller. If $\frac{-u_{k+1}^{2}+u_{k}^{2}}{2}>0$, i.e., the slope between $u_{k}^{2}$ and $u_{k+1}^{2}$ is negative, then the mesh interval $\delta_{k}$ becomes larger. Thus this scheme creates refined mesh grid for given data $\left\{x_{k}, u_{k}\right\}$ for $k=0,1,2, \cdots, N$, i.e., mesh grid is refined in which slopes are steep.

\section{Method 2:}

Step 1: The Lax pair of the SP equation is given by (10) with (13) and (14). This is transformed into (8) with (9) via the reciprocal (hodograph) transformation (4).

Step 2: By discretizing the Lax pair (8) with (9), we obtain the following linear $2 \times 2$ system (Lax pair):

$$
\Psi_{k+1}=U_{k} \Psi_{k}, \quad \frac{\partial \Psi_{k}}{\partial T}=V_{k} \Psi_{k}
$$

where

$$
\begin{aligned}
U_{k} & =\left(\begin{array}{cc}
1-\mathrm{i} \lambda a \rho_{k} & -\mathrm{i} \lambda \frac{u_{k+1}-u_{k}}{a} \\
-\mathrm{i} \lambda \frac{u_{k+1}-u_{k}}{a} & 1+\mathrm{i} \lambda a \rho_{k}
\end{array}\right), \\
V_{k} & =\left(\begin{array}{c}
\frac{\mathrm{i}}{4 \lambda}-\frac{u_{k}}{2} \\
\frac{u_{k}}{2}-\frac{1}{4 \lambda}
\end{array}\right),
\end{aligned}
$$

where $\Psi_{k}$ is a two-component vector. The compatibility condition yields the semi-discrete CD system (21) and (22).

Step 3: Consider a discrete analogue of the reciprocal (hodograph) transformation (23). Now we introduce the mesh interval $\delta_{k}=x_{k+1}-x_{k}$ which satisfies the relation $\delta_{k}=a \rho_{k}$, so one can rewrite $U_{k}$ and $V_{k}$ by using lattice intervals $\delta_{k}$ and replacing $\lambda$ by $i \lambda$ :

$$
\begin{aligned}
U_{k} & =\left(\begin{array}{cc}
1+\lambda \delta_{k} & \lambda \frac{u_{k+1}-u_{k}}{a} \\
\lambda \frac{u_{k+1}-u_{k}}{a} & 1-\lambda \delta_{k}
\end{array}\right), \\
V_{k} & =\left(\begin{array}{c}
\frac{1}{4 \lambda}-\frac{u_{k}}{2} \\
\frac{u_{k}}{2}-\frac{1}{4 \lambda}
\end{array}\right) .
\end{aligned}
$$

Step 4: This Lax pair provides (26) and (27) which is nothing but the self-adaptive moving mesh scheme for the SP equation.

Numerical simulations Here we show some examples of numerical simulations using the self-adaptive moving mesh scheme (26) and (27). As a time marching method, we use the improved Euler's method.

Multi-soliton solutions of the SP equation are given by

$$
\begin{aligned}
& u=\frac{g}{f}, \quad \rho=1-2(\ln f)_{X T}, \\
& x=X_{0}+\int_{X_{0}}^{X} \rho(\tilde{X}, T) d \tilde{X}, \quad t=T
\end{aligned}
$$

$f=\left|\begin{array}{cc}\mathcal{A}_{N} & I_{N} \\ -I_{N} & \mathcal{B}_{N}\end{array}\right|=\left|I_{N}+\mathcal{A}_{N} \mathcal{B}_{N}\right|$

$$
g=\left|\begin{array}{ccc}
\mathcal{A}_{N} & I_{N} & \mathbf{e}_{N}^{\top} \\
-I_{N} & \mathcal{B}_{N} & \mathbf{0}^{\top} \\
\mathbf{0} & -\mathbf{a}_{N} & 0
\end{array}\right|
$$


where

$$
\begin{gathered}
\mathcal{A}_{N}=\left(\begin{array}{ccccc}
\frac{\mathrm{e}^{\xi_{1}+\xi_{1}}}{4\left(1 / p_{1}+1 / p_{1}\right)} & \frac{\mathrm{e}^{\xi_{1}+\xi_{2}}}{4\left(1 / p_{1}+1 / p_{2}\right)} & \cdots & \frac{\mathrm{e}^{\xi_{1}+\xi_{N}}}{4\left(1 / p_{1}+1 / p_{N}\right)} \\
\frac{\mathrm{e}^{\xi_{2}+\xi_{1}}}{4\left(1 / p_{2}+1 / p_{1}\right)} & \frac{\mathrm{e}^{\xi_{2}+\xi_{2}}}{4\left(1 / p_{2}+1 / p_{2}\right)} & \cdots & \frac{\mathrm{e}^{\xi_{2}+\xi_{N}}}{4\left(1 / p_{2}+1 / p_{N}\right)} \\
\vdots & \vdots & \ddots & \vdots \\
\frac{\mathrm{e}^{\xi_{N}+\xi_{1}}}{4\left(1 / p_{N}+1 / p_{1}\right)} & \frac{\mathrm{e}^{\xi_{N}+\xi_{2}}}{4\left(1 / p_{N}+1 / p_{2}\right)} & \cdots & \frac{\mathrm{e}^{\xi_{N}+\xi_{N}}}{4\left(1 / p_{N}+1 / p_{N}\right)}
\end{array}\right), \\
\mathcal{B}_{N}=\left(\begin{array}{cccc}
\frac{a_{1} a_{1}}{1 / p_{1}+1 / p_{1}} & \frac{a_{1} a_{2}}{1 / p_{1}+1 / p_{2}} & \cdots & \frac{a_{1} a_{N}}{1 / p_{1}+1 / p_{N}} \\
\frac{a_{2} a_{1}}{1 / p_{2}+1 / p_{1}} & \frac{a_{2} a_{2}}{1 / p_{2}+1 / p_{2}} & \cdots & \frac{a_{2} a_{N}}{1 / p_{2}+1 / p_{N}} \\
\vdots & \vdots & \ddots & \vdots \\
\frac{a_{N} a_{1}}{1 / p_{N}+1 / p_{1}} & \frac{a_{N} a_{2}}{1 / p_{N}+1 / p_{2}} & \cdots & \frac{a_{N} a_{N}}{1 / p_{N}+1 / p_{N}}
\end{array}\right),
\end{gathered}
$$

and $I_{N}$ is the $N \times N$ identity matrix, $\mathbf{a}^{\top}$ is the transpose of $\mathbf{a}$,

$$
\begin{aligned}
& \mathbf{a}_{N}=\left(a_{1}, a_{2}, \cdots, a_{N}\right), \quad \mathbf{e}_{N}=\left(e^{\xi_{1}}, e^{\xi_{2}}, \cdots, e^{\xi_{N}}\right), \\
& \mathbf{0}=(0,0, \cdots, 0), \\
& \xi_{i}=p_{i} X+\frac{1}{p_{i}} T, \quad 1 \leq i \leq N .
\end{aligned}
$$

For example, the $\tau$-functions $f$ and $g$ of the 2 -soliton solution are written as

$$
\begin{aligned}
f= & 1+\frac{a_{1}^{2} p_{1}^{2}}{16} e^{2 \xi_{1}}+\frac{a_{2}^{2} p_{2}^{2}}{16} e^{2 \xi_{2}}+\frac{a_{1} a_{2} p_{1}^{2} p_{2}^{2}}{2\left(p_{1}+p_{2}\right)^{2}} e^{\xi_{1}+\xi_{2}} \\
& +\frac{a_{1}^{2} a_{2}^{2} p_{1}^{2} p_{2}^{2}}{256}\left(\frac{p_{1}-p_{2}}{p_{1}+p_{2}}\right)^{4} e^{2 \xi_{1}+2 \xi_{2}}
\end{aligned}
$$

$$
\begin{aligned}
g= & a_{1} e^{\xi_{1}}+a_{2} e^{\xi_{2}}+\frac{a_{1} a_{2}^{2} p_{2}^{2}}{16}\left(\frac{p_{1}-p_{2}}{p_{1}+p_{2}}\right)^{2} e^{\xi_{1}+2 \xi_{2}} \\
& +\frac{a_{1}^{2} a_{2} p_{1}^{2}}{16}\left(\frac{p_{1}-p_{2}}{p_{1}+p_{2}}\right)^{2} e^{2 \xi_{1}+\xi_{2}}
\end{aligned}
$$

where

$$
\xi_{i}=p_{i} X+\frac{1}{p_{i}} T, \quad i=1,2 .
$$

There are two types of 2-loop soliton solutions and a type of breather solutions:

(1) Interactions of two loop solitons if both $a_{1}$ and $a_{2}$ are positive, or if both $a_{1}$ and $a_{2}$ are negative. The wave numbers $p_{1}$ and $p_{2}$ are chosen real.

(2) Interactions of a loop soliton and an anti-loop soliton if $a_{1}$ and $a_{2}$ have opposite signs. The wave numbers $p_{1}$ and $p_{2}$ are chosen real.

(3) Breather solutions if the wave numbers $p_{1}$ and $p_{2}$ are chosen complex and satisfy $p_{2}=p_{1}^{*}$ and $a_{2}=a_{1}^{*}$ in the above $\tau$-functions.

Here we show three examples of numerical simulations of the SP equation. We use the number of mesh grid points $N=200$, the width of the computational domain $\mathrm{D}=80$, and the time interval $d t=0.0001$. Figure 1 shows the numerical simulation of the 2-loop soliton solution of the SP equation. Figure 2 shows the numerical simulation of the solution describing the interaction of a loop soliton and anti-loop soliton of the SP equation. In Figure 3, we show the numerical simulation of the breather solution of the SP equation. In these three examples, numerical results have good agreement with exact solutions of the $\mathrm{SP}$ equation. It is possible to have more accurate numerical results if we increase the number of mesh grid points and use smaller time steps.

\section{Self-adaptive moving mesh schemes for the coupled short pulse equation and the complex short pulse equation}

By means of the above methods (Method 1 or Method 2) for constructing self-adaptive moving mesh schemes, we can also construct self-adaptive moving mesh schemes for the coupld SP equation and the complex SP equation. Here we show only the results obtained by using Method 1 and Method 2. Note that both methods give the same results.

Consider the following linear $2 \times 2$ system:

$$
\frac{\partial \Psi}{\partial X}=U \Psi, \quad \frac{\partial \Psi}{\partial T}=V \Psi,
$$

where

$$
U=-\mathrm{i} \lambda\left(\begin{array}{cc}
\rho & u_{X} \\
v_{X} & -\rho
\end{array}\right), \quad V=\left(\begin{array}{cc}
\frac{\mathrm{i}}{4 \lambda} & -\frac{u}{2} \\
\frac{\nu}{2} & -\frac{\mathrm{i}}{4 \lambda}
\end{array}\right),
$$

where $\Psi$ is a two-component vector. The compatibility condition yields the coupled CD system $[24,25]$

$$
\begin{aligned}
& \frac{\partial \rho}{\partial T}-\frac{\partial}{\partial X}\left(-\frac{u v}{2}\right)=0, \\
& \frac{\partial^{2} u}{\partial X \partial T}=\rho u, \\
& \frac{\partial^{2} v}{\partial X \partial T}=\rho v .
\end{aligned}
$$

Note the coupled CD system (45), (46) and (47) can be transformed into the bilinear equations

$$
\begin{aligned}
& D_{T}^{2} f \cdot f=\frac{1}{2} g h, \\
& D_{X} D_{T} f \cdot g=f g, \\
& D_{X} D_{T} f \cdot h=f h,
\end{aligned}
$$

via the dependent variable transformation

$$
u=\frac{g}{f}, \quad v=\frac{h}{f}, \quad \rho=1-2(\ln f)_{X T} .
$$

Applying the reciprocal (hodograph) transformation (4) into the above linear problem (43) and (44), we obtain the linear $2 \times 2$ system (Lax pair) for the coupled SP equation [31]:

$$
\frac{\partial \Psi}{\partial x}=\tilde{U} \Psi, \quad \frac{\partial \Psi}{\partial t}=\tilde{V} \Psi
$$




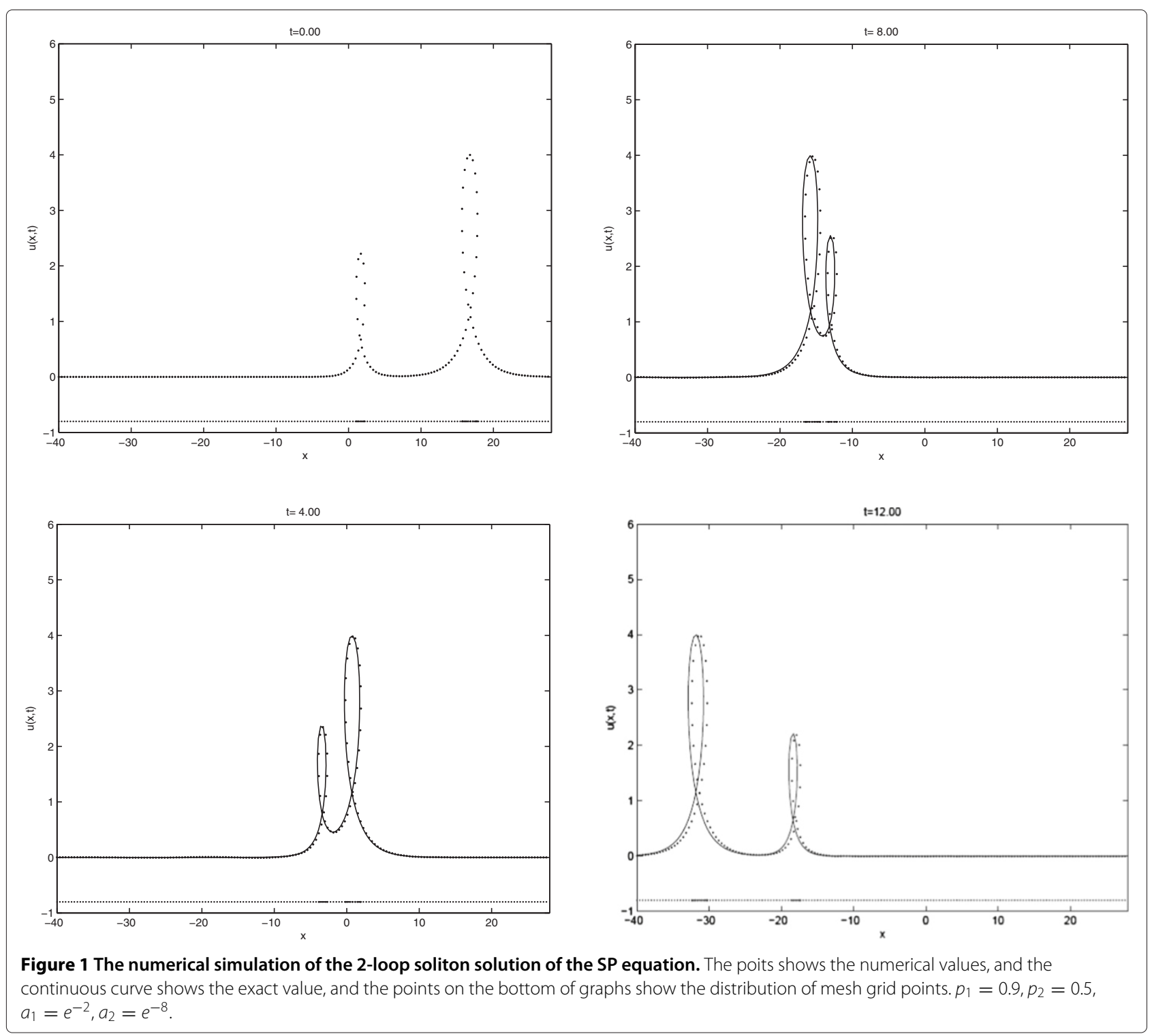

where

$$
\begin{aligned}
& \tilde{U}=-\mathrm{i} \lambda\left(\begin{array}{cc}
1 & u_{x} \\
v_{x} & -1
\end{array}\right), \\
& \tilde{V}=\left(\begin{array}{c}
\frac{\mathrm{i}}{4 \lambda}-\frac{\mathrm{i} \lambda}{2} u \nu-\frac{u}{2}-\frac{\mathrm{i} \lambda}{2} u v u_{x} \\
\frac{v}{2}-\frac{\mathrm{i} \lambda}{2} u \nu v_{x}-\frac{\mathrm{i}}{4 \lambda}+\frac{\mathrm{i} \lambda}{2} u v
\end{array}\right),
\end{aligned}
$$

which can be rewritten as

$$
\begin{aligned}
& \tilde{U}=\lambda\left(\begin{array}{cc}
1 & u_{x} \\
v_{x} & -1
\end{array}\right) \\
& \tilde{V}=\left(\begin{array}{cc}
\frac{1}{4 \lambda}+\frac{\lambda}{2} u v & -\frac{u}{2}+\frac{\lambda}{2} u v u_{x} \\
\frac{v}{2}+\frac{\lambda}{2} u v v_{x} & -\frac{1}{4 \lambda}-\frac{\lambda}{2} u v
\end{array}\right),
\end{aligned}
$$

by replacing $\lambda$ by $i \lambda$. The compatibility condition yields the coupled SP equation $[9,31]$

$$
\begin{aligned}
& u_{x t}=u+\frac{1}{2}\left(u v u_{x}\right)_{x}, \\
& v_{x t}=v+\frac{1}{2}\left(u \nu v_{x}\right)_{x} .
\end{aligned}
$$

Letting $u$ be a complex function and $v=u^{*}$ where $u^{*}$ is a complex conjugate of $u$, the compatibility condition of the above linear $2 \times 2$ systems yields the complex CD system [26,30]

$$
\begin{aligned}
& \frac{\partial \rho}{\partial T}-\frac{\partial}{\partial X}\left(-\frac{|u|^{2}}{2}\right)=0, \\
& \frac{\partial^{2} u}{\partial X \partial T}=\rho u,
\end{aligned}
$$




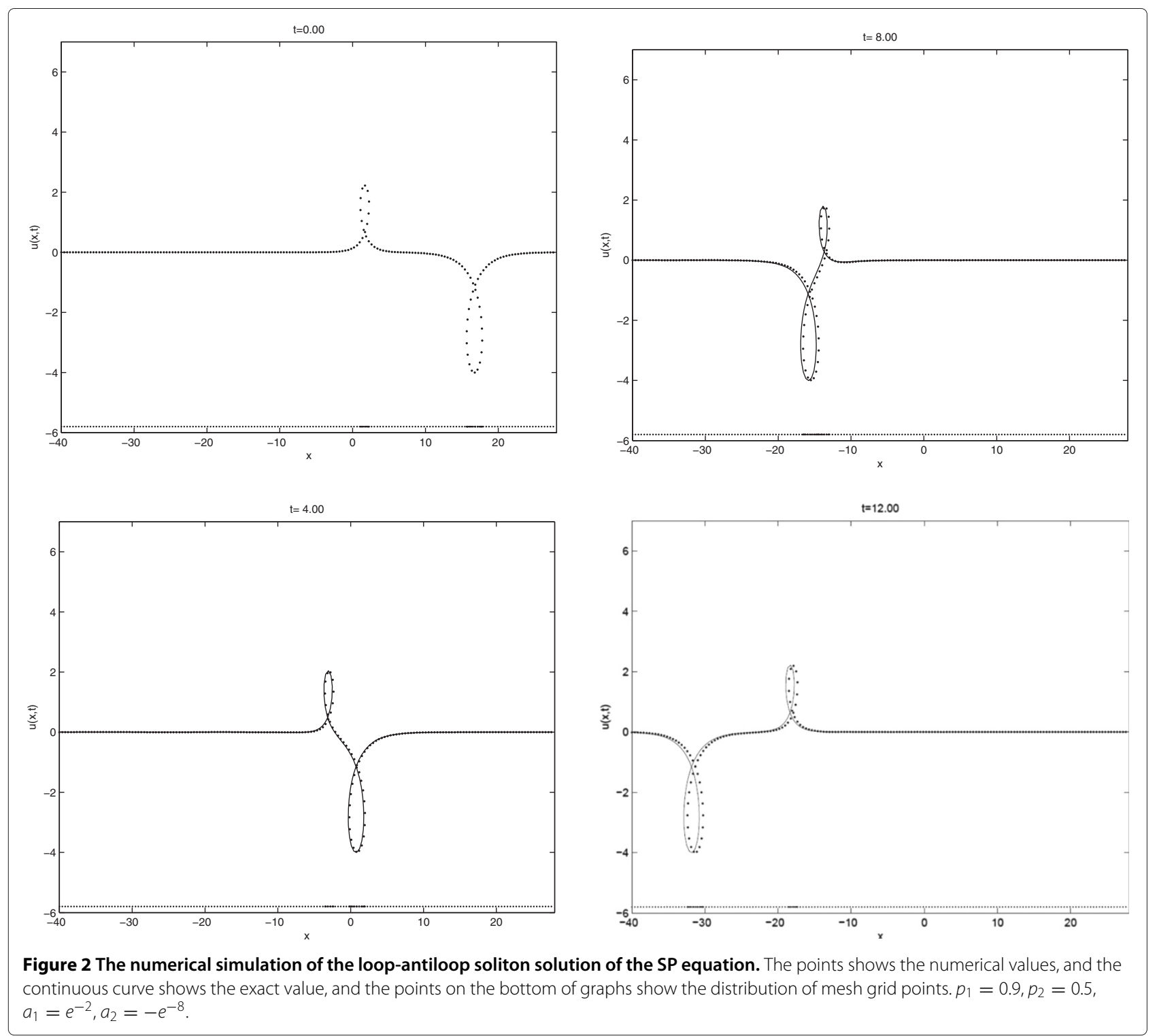

$$
\frac{\partial^{2} u^{*}}{\partial X \partial T}=\rho u^{*}
$$

and the complex SP equation [10]

$$
\begin{aligned}
& u_{x t}=u+\frac{1}{2}\left(|u|^{2} u_{x}\right)_{x}, \\
& u_{x t}^{*}=u^{*}+\frac{1}{2}\left(|u|^{2} u_{x}^{*}\right)_{x} .
\end{aligned}
$$

Note the complex CD system (59), (60) and (61) can be transformed into the bilinear equations

$$
\begin{aligned}
& D_{T}^{2} f \cdot f=\frac{1}{2} g g^{*}, \\
& D_{X} D_{T} f \cdot g=f g, \\
& D_{X} D_{T} f \cdot g^{*}=f g^{*},
\end{aligned}
$$

via the dependent variable transformation

$$
u=\frac{g}{f}, \quad u^{*}=\frac{g^{*}}{f}, \quad \rho=1-2(\ln f)_{X T} .
$$

Using the dependent variables $u^{(R)}$ and $u^{(\mathrm{I})}$ such that $u^{(R)}=\operatorname{Re} u, u^{(\mathrm{I})}=\operatorname{Im} u$, the complex SP equation can be written as

$$
\begin{aligned}
& u_{x t}^{(R)}=u^{(R)}+\frac{1}{2}\left(\left(u^{(R)^{2}}+u^{(\mathrm{I})^{2}}\right) u_{x}^{(R)}\right)_{x}, \\
& u_{x t}^{(\mathrm{I})}=u^{(\mathrm{I})}+\frac{1}{2}\left(\left(u^{(R)^{2}}+u^{(\mathrm{I})^{2}}\right) u_{x}^{(\mathrm{I})}\right)_{x} .
\end{aligned}
$$




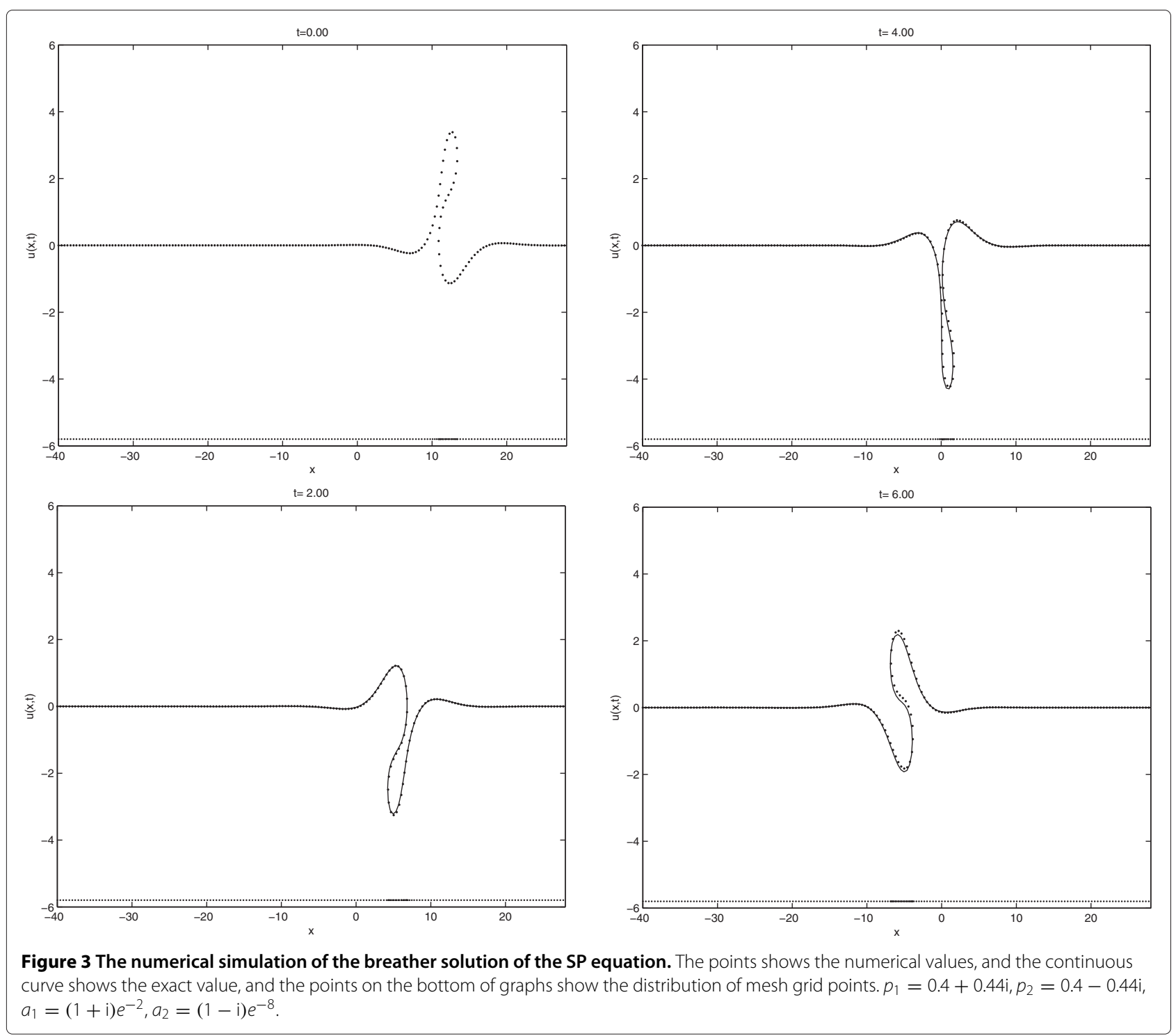

Consider the following linear $2 \times 2$ system (32) with

$$
\begin{aligned}
U_{k} & =\left(\begin{array}{cc}
1-\mathrm{i} \lambda a \rho_{k} & -\mathrm{i} \lambda \frac{u_{k+1}-u_{k}}{a} \\
-\mathrm{i} \lambda \frac{v_{k+1}-v_{k}}{a} & 1+\mathrm{i} \lambda a \rho_{k}
\end{array}\right), \\
V_{k} & =\left(\begin{array}{c}
\frac{\mathrm{i}}{4 \lambda}-\frac{u_{k}}{2} \\
\frac{v_{k}}{2}-\frac{1}{4 \lambda}
\end{array}\right),
\end{aligned}
$$

where $\Psi_{k}$ is a two-component vector. The compatibility condition (32) with (70) and (71) yields the semi-discrete coupled CD system

$$
\partial_{T} \rho_{k}-\frac{\left(-\frac{u_{k+1} v_{k+1}}{2}\right)-\left(-\frac{u_{k} v_{k}}{2}\right)}{a}=0,
$$

$$
\begin{aligned}
& \partial_{T}\left(\frac{u_{k+1}-u_{k}}{a}\right)=\rho_{k} \frac{u_{k+1}+u_{k}}{2}, \\
& \partial_{T}\left(\frac{v_{k+1}-v_{k}}{a}\right)=\rho_{k} \frac{v_{k+1}+v_{k}}{2} .
\end{aligned}
$$

We can rewrite $U_{k}$ and $V_{k}$ by using lattice intervals $\delta_{k}(=$ $\left.a \rho_{k}=x_{k+1}-x_{k}\right)$ and replacing $\lambda$ by $\mathrm{i} \lambda$ :

$$
\begin{aligned}
U_{k} & =\left(\begin{array}{cc}
1+\lambda \delta_{k} & \lambda \frac{u_{k+1}-u_{k}}{a} \\
\lambda \frac{v_{k+1}-v_{k}}{a} & 1-\lambda \delta_{k}
\end{array}\right), \\
V_{k} & =\left(\begin{array}{c}
\frac{1}{4 \lambda}-\frac{u_{k}}{2} \\
\frac{v_{k}}{2}-\frac{1}{4 \lambda}
\end{array}\right) .
\end{aligned}
$$


The compatibility condition of (32) with (75) and (76) provides the self-adaptive moving mesh scheme for the coupled SP equation

$$
\begin{aligned}
& \partial_{T} \delta_{k}=\frac{-u_{k+1} v_{k+1}+u_{k} v_{k}}{2}, \\
& \partial_{T}\left(u_{k+1}-u_{k}\right)=\delta_{k} \frac{u_{k+1}+u_{k}}{2}, \\
& \partial_{T}\left(v_{k+1}-v_{k}\right)=\delta_{k} \frac{v_{k+1}+v_{k}}{2},
\end{aligned}
$$

where $x_{k}=X_{k}+\sum_{j=0}^{k-1} \delta_{j}$ and $\delta_{k}=x_{k+1}-x_{k}, x_{0}=X_{0}$.

The discrete reciprocal (hodograph) transformation $x_{k}=X_{k}+\sum_{j=0}^{k-1} \delta_{j}$ yields

$$
\begin{aligned}
\frac{\Delta}{\Delta X_{k}} & =\frac{\Delta}{a}=\frac{\Delta x_{k}}{a} \frac{\Delta}{\Delta x_{k}}=\rho_{k} \frac{\Delta}{\Delta x_{k}}=\rho_{k} \frac{\Delta}{\delta_{k}}, \\
\frac{\partial}{\partial T} & =\frac{\partial}{\partial t}+\frac{\partial x_{k}}{\partial T} \frac{\partial}{\partial x_{k}}=\frac{\partial}{\partial t}+\sum_{j=0}^{k-1} \frac{\partial \delta_{j}}{\partial T} \frac{\partial}{\partial x_{k}} \\
& =\frac{\partial}{\partial t}+\left(\sum_{j=0}^{k-1} \frac{-u_{k+1} v_{k+1}+u_{k} v_{k}}{2}\right) \frac{\partial}{\partial x_{k}} \\
& =\frac{\partial}{\partial t}+\left(\frac{-u_{k+1} v_{k+1}+u_{0} v_{0}}{2}\right) \frac{\partial}{\partial x_{k}} \\
& =\frac{\partial}{\partial t}+\left(\frac{-u_{k+1} v_{k+1}}{2}\right) \frac{\partial}{\partial x_{k}},
\end{aligned}
$$

if $u_{0}=0, v_{0}=0$.

Applying this to eqs. (78) and (79), we obtain

$$
\begin{aligned}
\frac{1}{\delta_{k}} \frac{\partial\left(u_{k+1}-u_{k}\right)}{\partial t} & -\frac{u_{k+1} v_{k+1}}{2} \frac{1}{\delta_{k}} \frac{\partial\left(u_{k+1}-u_{k}\right)}{\partial x_{k}} \\
& =\frac{u_{k+1}+u_{k}}{2}, \\
\frac{1}{\delta_{k}} \frac{\partial\left(v_{k+1}-v_{k}\right)}{\partial t} & -\frac{u_{k+1} v_{k+1}}{2} \frac{1}{\delta_{k}} \frac{\partial\left(v_{k+1}-v_{k}\right)}{\partial x_{k}} \\
& =\frac{v_{k+1}+v_{k}}{2} .
\end{aligned}
$$

In the continuous limit $\delta_{k} \rightarrow 0$, this leads to the coupled SP equation (57) and (58).

Note that the semi-discrete coupled CD system and the self-adaptive moving mesh scheme for the coupled SP equation can be transformed into the bilinear equations

$$
\begin{aligned}
& D_{T}^{2} f_{k} \cdot f_{k}=\frac{1}{2} g_{k} h_{k}, \\
& \frac{1}{a} D_{T}\left(f_{k+1} \cdot g_{k}-f_{k} \cdot g_{k+1}\right)=\frac{1}{2}\left(f_{k+1} g_{k}+f_{k} g_{k+1}\right), \\
& \frac{1}{a} D_{T}\left(f_{k+1} \cdot h_{k}-f_{k} \cdot h_{k+1}\right)=\frac{1}{2}\left(f_{k+1} h_{k}+f_{k} h_{k+1}\right),
\end{aligned}
$$

via the dependent variable transformation

$$
\begin{aligned}
& u_{k}=\frac{g_{k}}{f_{k}}, \quad v_{k}=\frac{h_{k}}{f_{k}}, \\
& \rho_{k}=\frac{\delta_{k}}{a}=1-\frac{2}{a}\left(\ln \frac{f_{k+1}}{f_{k}}\right)_{T} .
\end{aligned}
$$

By letting $u_{k}$ be a complex function and adding a constraint $v_{k}=u_{k}^{*}$, we obtain the semi-discrete complex CD system

$$
\begin{aligned}
& \partial_{T} \rho_{k}-\frac{\left(-\frac{u_{k+1} v_{k+1}}{2}\right)-\left(-\frac{\left|u_{k}\right|^{2}}{2}\right)}{a}=0, \\
& \partial_{T}\left(\frac{u_{k+1}-u_{k}}{a}\right)=\rho_{k} \frac{u_{k+1}+u_{k}}{2}, \\
& \partial_{T}\left(\frac{u_{k+1}^{*}-u_{k}^{*}}{a}\right)=\rho_{k} \frac{u_{k+1}^{*}+u_{k}^{*}}{2},
\end{aligned}
$$

and the self-adaptive moving mesh scheme for the complex SP equation

$$
\begin{aligned}
& \partial_{T} \delta_{k}=\frac{-\left|u_{k+1}\right|^{2}+\left|u_{k}\right|^{2}}{2}, \\
& \partial_{T}\left(u_{k+1}-u_{k}\right)=\delta_{k} \frac{u_{k+1}+u_{k}}{2}, \\
& \partial_{T}\left(u_{k+1}^{*}-u_{k}^{*}\right)=\delta_{k} \frac{u_{k+1}^{*}+u_{k}^{*}}{2} .
\end{aligned}
$$

Note that the semi-discrete complex CD system and the self-adaptive moving mesh scheme for the complex SP equation can be transformed into the bilinear equations

$$
\begin{aligned}
& D_{T}^{2} f_{k} \cdot f_{k}=\frac{1}{2} g_{k} g_{k}^{*} \\
& \frac{1}{a} D_{T}\left(f_{k+1} \cdot g_{k}-f_{k} \cdot g_{k+1}\right)=\frac{1}{2}\left(f_{k+1} g_{k}+f_{k} g_{k+1}\right),
\end{aligned}
$$

$$
\frac{1}{a} D_{T}\left(f_{k+1} \cdot g_{k}^{*}-f_{k} \cdot g_{k+1}^{*}\right)=\frac{1}{2}\left(f_{k+1} g_{k}^{*}+f_{k} g_{k+1}^{*}\right),
$$

via the dependent variable transformation

$$
\begin{aligned}
& u_{k}=\frac{g_{k}}{f_{k}}, \quad u_{k}=\frac{g_{k}^{*}}{f_{k}}, \\
& \rho_{k}=\frac{\delta_{k}}{a}=1-\frac{2}{a}\left(\ln \frac{f_{k+1}}{f_{k}}\right)_{T} .
\end{aligned}
$$


Using the dependent variables $u_{k}^{(R)}$ and $u_{k}^{(\mathrm{I})}$ such that $u_{k}^{(R)}=\operatorname{Re} u_{k}, u_{k}^{(\mathrm{I})}=\operatorname{Im} u_{k}$, the complex SP equation can be written as

$$
\begin{array}{r}
\partial_{T} \delta_{k} \\
=-\frac{1}{2}\left(\left(u_{k+1}^{(R)}\right)^{2}+\left(u_{k+1}^{(I)}\right)^{2}\right) \\
+\frac{1}{2}\left(\left(u_{k+1}^{(R)}\right)^{2}+\left(u_{k+1}^{(I)}\right)^{2}\right), \\
\partial_{T}\left(u_{k+1}^{(R)}-u_{k}^{(R)}\right)=\delta_{k} \frac{u_{k+1}^{(R)}+u_{k}^{(R)}}{2}, \\
\partial_{T}\left(u_{k+1}^{(I)}-u_{k}^{(I)}\right)=\delta_{k} \frac{u_{k+1}^{(I)}+u_{k}^{(I)}}{2} .
\end{array}
$$

We remark that the discretization of the generalized CD systems were proposed by Vinet and Yu recently $[41,42]$. Our results are consistent with their results.

Numerical simulations Here we show some examples of numerical simulations of the complex SP equation using the self-adaptive moving mesh scheme (91), (92) and (93). As a time marching method, we use the improved Euler's method.

The multi-soliton solutions of the complex SP equation (62) and (63) are given by the following formula:

$$
\begin{aligned}
& u=\frac{g}{f}, \quad u^{*}=\frac{g^{*}}{f}, \quad \rho=1-2(\ln f)_{X T}, \\
& x=X_{0}+\int_{X_{0}}^{X} \rho(\tilde{X}, T) d \tilde{X}, \quad t=T,
\end{aligned}
$$

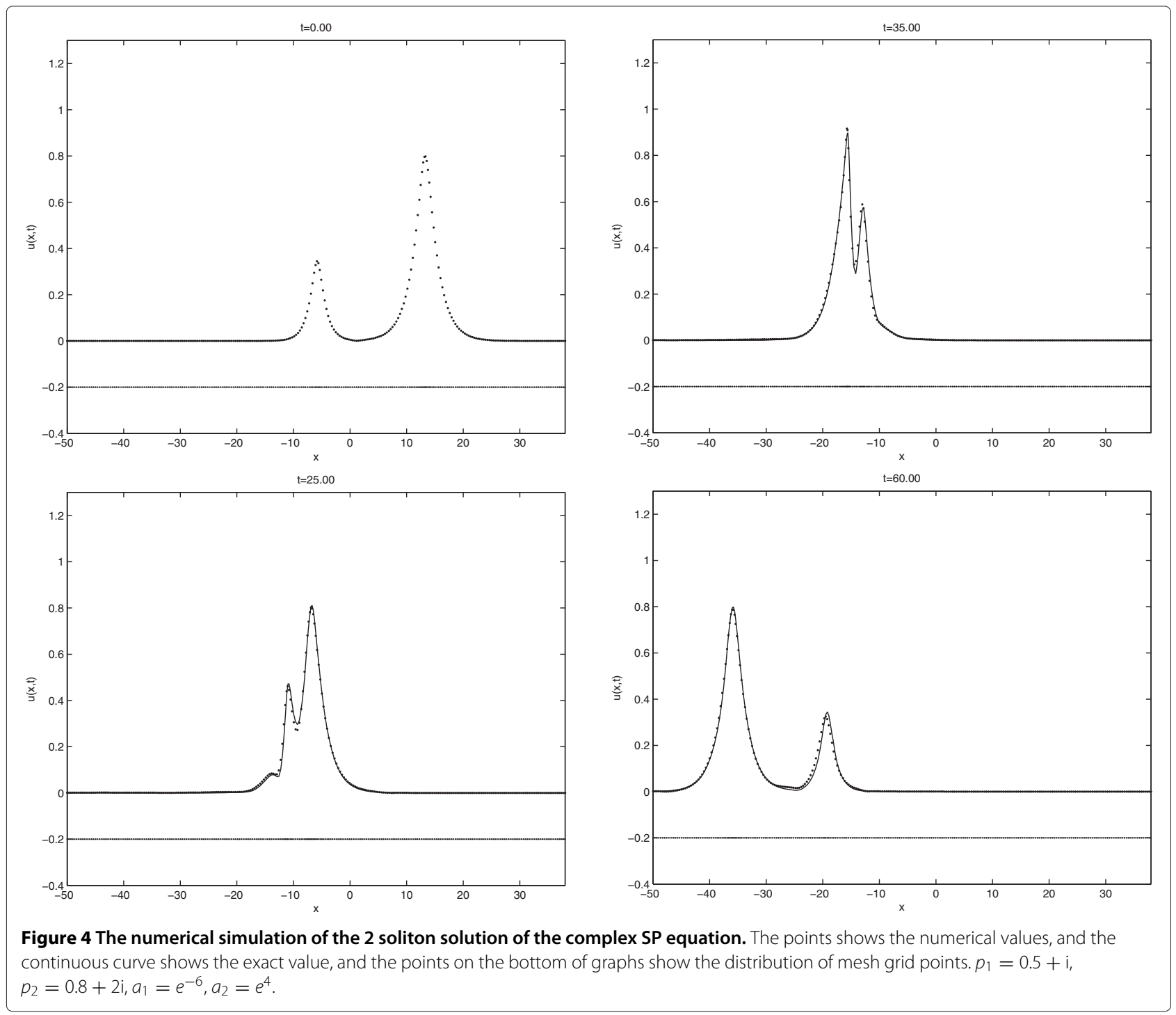




$$
\begin{aligned}
& f=\left|\begin{array}{cc}
\mathcal{A}_{N} & I_{N} \\
-I_{N} & \mathcal{B}_{N}
\end{array}\right|=\left|I_{N}+\mathcal{A}_{N} \mathcal{B}_{N}\right| \\
& g=\left|\begin{array}{ccc}
\mathcal{A}_{N} & I_{N} & \mathbf{e}_{N}^{\top} \\
-I_{N} & \mathcal{B}_{N} & \mathbf{0}^{\top} \\
\mathbf{0} & -\mathbf{a}_{N} & 0
\end{array}\right| \\
& g^{*}=\left|\begin{array}{ccc}
\mathcal{A}_{N} & I_{N} & \mathbf{0}^{\top} \\
-I_{N} & \mathcal{B}_{N} & \mathbf{a}_{N}^{*} \\
\mathbf{e}_{N}^{*} & \mathbf{0} & 0
\end{array}\right|
\end{aligned}
$$

where

$$
\mathcal{A}_{N}=\left(\begin{array}{cccc}
\frac{\mathrm{e}^{\xi_{1}+\xi_{1}^{*}}}{4\left(1 / p_{1}+1 / p_{1}^{*}\right)} & \frac{\mathrm{e}^{\xi_{1}+\xi_{2}^{*}}}{4\left(1 / p_{1}+1 / p_{2}^{*}\right)} & \cdots & \frac{\mathrm{e}^{\xi_{1}+\xi_{N}^{*}}}{4\left(1 / p_{1}+1 / p_{N}^{*}\right)} \\
\frac{\mathrm{e}^{\xi_{2}+\xi_{1}^{*}}}{4\left(1 / p_{2}+1 / p_{1}^{*}\right)} & \frac{\mathrm{e}^{\xi_{2}+\xi_{2}^{*}}}{4\left(1 / p_{2}+1 / p_{2}^{*}\right)} & \cdots & \frac{\mathrm{e}^{\xi_{2}+\xi_{N}^{*}}}{4\left(1 / p_{2}+1 / p_{N}^{*}\right)} \\
\vdots & \vdots & \ddots & \vdots \\
\frac{\mathrm{e}^{\xi_{N}+\xi_{1}^{*}}}{4\left(1 / p_{N}+1 / p_{1}^{*}\right)} & \frac{\mathrm{e}^{\xi_{N}+\xi_{2}^{*}}}{4\left(1 / p_{N}+1 / p_{2}^{*}\right)} & \cdots & \frac{\mathrm{e}^{\xi_{N}+\xi_{N}^{*}}}{4\left(1 / p_{N}+1 / p_{N}^{*}\right)}
\end{array}\right)
$$

$$
\mathcal{B}_{N}=\left(\begin{array}{cccc}
\frac{a_{1} a_{1}^{*}}{1 / p_{1}+1 / p_{1}^{*}} & \frac{a_{2} a_{1}^{*}}{1 / p_{2}+1 / p_{1}^{*}} & \cdots & \frac{a_{N} a_{1}^{*}}{1 / p_{N}+1 / p_{1}^{*}} \\
\frac{a_{1} a_{2}^{*}}{1 / p_{1}+1 / p_{2}^{*}} & \frac{a_{2} a_{2}^{*}}{1 / p_{2}+1 / p_{2}^{*}} & \cdots & \frac{a_{N} a_{2}^{*}}{1 / p_{N}+1 / p_{2}^{*}} \\
\vdots & \vdots & \ddots & \vdots \\
\frac{a_{1} a_{N}^{*}}{1 / p_{1}+1 / p_{N}^{*}} & \frac{a_{2} a_{N}^{*}}{1 / p_{2}+1 / p_{N}^{*}} & \cdots & \frac{a_{N} a_{N}^{*}}{1 / p_{N}+1 / p_{N}^{*}}
\end{array}\right)
$$

and $I_{N}$ is the $N \times N$ identity matrix, $\mathbf{a}^{\top}$ is the transpose of $\mathbf{a}$,

$$
\begin{aligned}
& \mathbf{a}_{N}=\left(a_{1}, a_{2}, \cdots, a_{N}\right), \quad \mathbf{e}_{N}=\left(e^{\xi_{1}}, e^{\xi_{2}}, \cdots, e^{\xi_{N}}\right), \\
& \mathbf{a}_{N}^{*}\left(a_{1}^{*}, a_{2}^{*}, \cdots, a_{N}^{*}\right), \quad \mathbf{e}_{N}^{*}=\left(e^{\xi_{1}^{*}}, e^{\xi_{2}^{*}}, \cdots, e^{\xi_{N}^{*}}\right), \\
& \mathbf{0}=(0,0, \cdots, 0), \\
& \xi_{i}=p_{i} X+\frac{1}{p_{i}} T, \quad \xi_{i}^{*}=p_{i}^{*} X+\frac{1}{p_{i}^{*}} T, \quad 1 \leq i \leq N .
\end{aligned}
$$
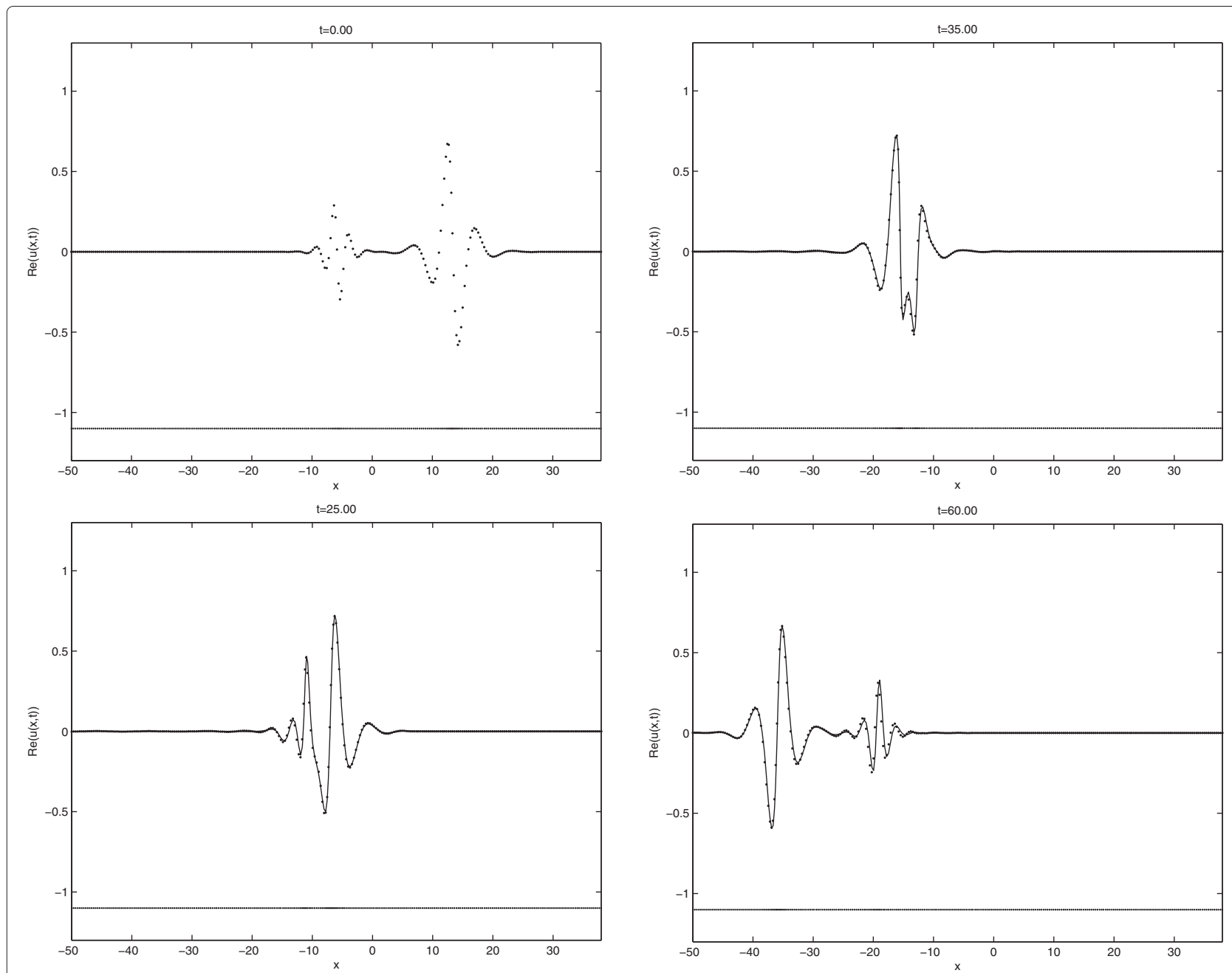

Figure 5 The numerical simulation of the 2 soliton solution of the complex SP equation. The graphs show the real part of $u$ of the complex SP equation. The points shows the numerical values, and the continuous curve shows the exact value, and the points on the bottom of graphs show the distribution of mesh grid points. $p_{1}=0.5+\mathrm{i}, p_{2}=0.8+2 \mathrm{i}, a_{1}=e^{-6}, a_{2}=e^{4}$. 
Fen et al. Pacific Journal of Mathematics for Industry 2014, 6:8

Page 12 of 14

http://www.pacific-mathforindustry.com/content/6/1/8

Note that this formula is obtained from the gram type determinant solution of the coupled SP equation in Appendix.

For example, the $\tau$-functions $f, g$ and $g^{*}$ of the 2 -soliton solution of the complex SP equation are written as

$$
\begin{aligned}
f= & +\frac{a_{1} a_{1}^{*} p_{1}^{2} p_{1}^{* 2}}{4\left(p_{1}+p_{1}^{*}\right)^{2}} e^{\xi_{1}+\xi_{1}^{*}}+\frac{a_{1} a_{2}^{*} p_{1}^{2} p_{2}^{* 2}}{4\left(p_{1}+p_{2}^{*}\right)^{2}} e^{\xi_{1}+\xi_{2}^{*}} \\
+ & \frac{a_{2} a_{1}^{*} p_{2}^{2} p_{1}^{* 2}}{4\left(p_{2}+p_{1}^{*}\right)^{2}} e^{\xi_{2}+\xi_{1}^{*}}+\frac{a_{2} a_{2}^{*} p_{2}^{2} p_{2}^{* 2}}{4\left(p_{2}+p_{2}^{*}\right)^{2}} e^{\xi_{2}+\xi_{2}^{*}} \\
+ & \frac{a_{1} a_{1}^{*} a_{2} a_{2}^{*} p_{1}^{2} p_{1}^{* 2} p_{2}^{2} p_{2}^{* 2}\left(p_{1}-p_{2}\right)^{2}\left(p_{1}^{*}-p_{2}^{*}\right)^{2}}{16\left(p_{1}+p_{1}^{*}\right)^{2}\left(p_{1}+p_{2}^{*}\right)^{2}\left(p_{2}+p_{1}^{*}\right)^{2}\left(p_{2}+p_{2}^{*}\right)^{2}} \\
& \times e^{\xi_{1}+\xi_{2}+\xi_{1}^{*}+\xi_{2}^{*}}
\end{aligned}
$$
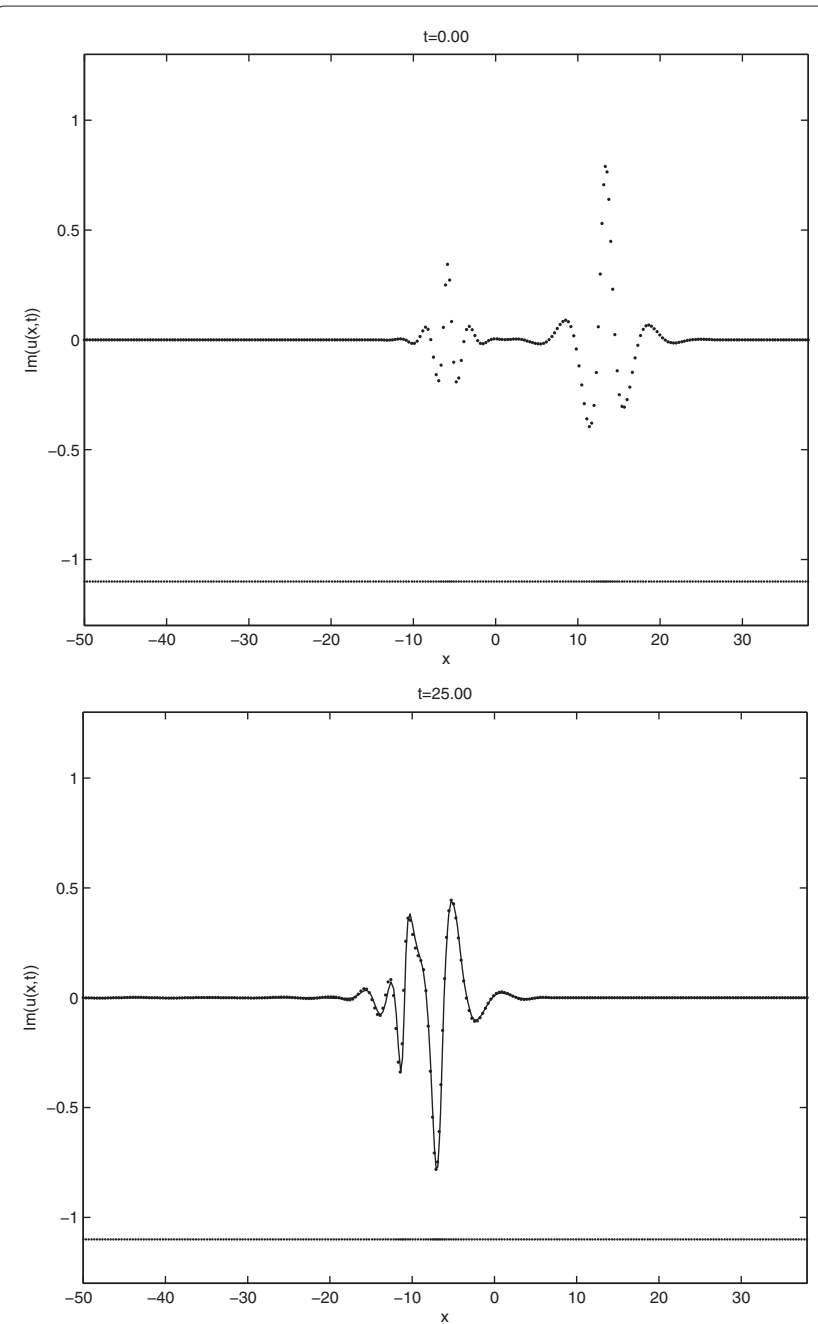

$$
\begin{aligned}
& g=a_{1} e^{\xi_{1}}+a_{2} e^{\xi_{2}}+\frac{a_{1} a_{2} a_{2}^{*}\left(p_{1}-p_{2}\right)^{2} p_{2}^{* 4}}{4\left(p_{1}+p_{2}^{*}\right)^{2}\left(p_{2}+p_{2}^{*}\right)^{2}} e^{\xi_{1}+\xi_{2}+\xi_{2}^{*}} \\
& +\frac{a_{1} a_{1}^{*} a_{2}\left(p_{1}-p_{2}\right)^{2} p_{1}^{* 4}}{4\left(p_{1}+p_{1}^{*}\right)^{2}\left(p_{2}+p_{1}^{*}\right)^{2}} e^{\xi_{1}+\xi_{1}^{*}+\xi_{2}}, \\
& g^{*}=a_{1}^{*} e^{\xi_{1}^{*}}+a_{2}^{*} e^{\xi_{2}^{*}}+\frac{a_{1}^{*} a_{2} a_{2}^{*}\left(p_{1}^{*}-p_{2}^{*}\right)^{2} p_{2}^{4}}{4\left(p_{1}^{*}+p_{2}\right)^{2}\left(p_{2}+p_{2}^{*}\right)^{2}} e^{\xi_{1}^{*}+\xi_{2}+\xi_{2}^{*}} \\
& +\frac{a_{1} a_{1}^{*} a_{2}^{*}\left(p_{1}^{*}-p_{2}^{*}\right)^{2} p_{1}^{4}}{4\left(p_{1}^{*}+p_{1}\right)^{2}\left(p_{1}+p_{2}^{*}\right)^{2}} e^{\xi_{1}+\xi_{1}^{*}+\xi_{2}^{*}},
\end{aligned}
$$

where

$$
\xi_{i}=p_{i} X+\frac{1}{p_{i}} T, \quad i=1,2 .
$$
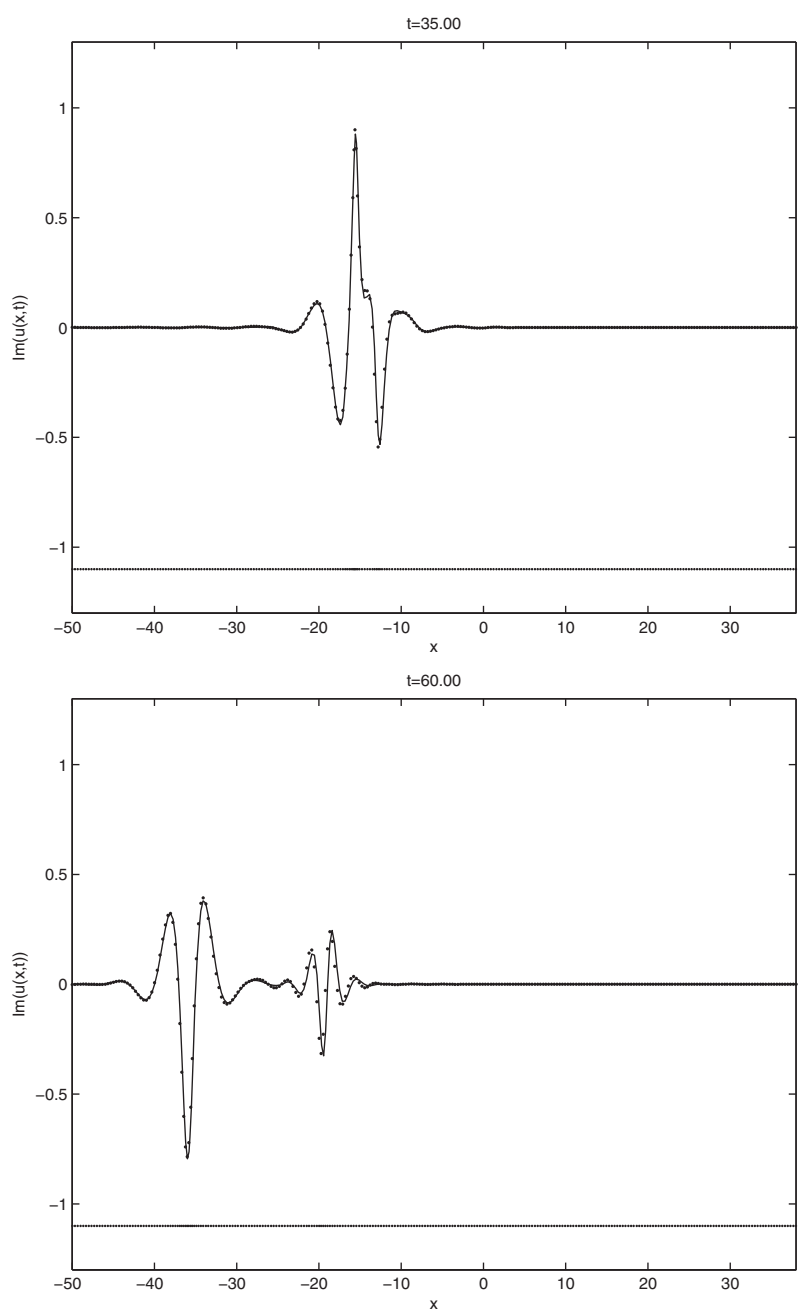

Figure 6 The numerical simulation of the 2 soliton solution of the complex SP equation. The graphs show the imaginary part of $u$ of the complex SP equation. The points shows the numerical values, and the continuous curve shows the exact value, and the points on the bottom of graphs show the distribution of mesh grid points. $p_{1}=0.5+\mathrm{i}, p_{2}=0.8+2 \mathrm{i}, a_{1}=e^{-6}, a_{2}=e^{4}$. 
Figure 4 shows the numerical simulation of the 2 soliton solution of the complex SP equation (62) and (63) by means of the self-adaptive moving mesh scheme (91), (92), and (93). Figures 5 and 6 show the graphs of the real part and the imaginary part of $u$ of the complex SP equation (62) and (63), respectively. Thus these graphs are the solution of equations (68) and (69). We use the number of mesh grid points $N=300$, the width of the computational domain $\mathrm{D}=100$, and the time interval $d t=$ 0.00005 . Again, the numerical result has good agreement with the exact solution of the complex SP equation.

\section{Concluding remarks}

We have proposed two systematic methods for constructing self-adaptive moving mesh schemes for a class of nonlinear wave equations which are transformed into a different class of nonlinear wave equations by reciprocal (hodograph) transformations. We have demonstrated how to create self-adaptive moving mesh schemes for short pulse type equations which are transformed into coupled dispersionless type systems by a reciprocal (hodograph) transformation. Self-adaptive moving mesh schemes have exact solutions such as multi-soliton solutions and Lax pairs, thus those schemes are integrable. Self-adaptive moving mesh schemes consist of two semidiscrete equations in which the time is continuous and the space is discrete. In self-adaptive moving mesh schemes, one of two equations is an evolution equation of mesh intervals which is deeply related to a discrete analogue of a reciprocal (hodograph) transformation. An evolution equations of mesh intervals is a discrete analogue of a conservation law of an original equation, and a set of mesh intervals corresponds to a conserved density which play a key role in generation of adaptive moving mesh. We have shown several examples of numerical computations of the short pulse type equations by using self-adaptive moving mesh schemes.

In our previous papers, we have investigated how to discretize the Camassa-Holm [13,34], the Hunter-Saxton [35], the short pulse [11,14], the WKI elastic beam [11], the Dym equation $[12,14]$ by using bilinear methods or by using a geometric approach. Based on our previous studies, we have proposed two systematic methods in sophisticated forms, one uses bilinear equations and another uses Lax pairs, for producing self-adaptive moving mesh schemes. Although we have discussed only short pulse type equations in this paper, our methods can be used to construct self-adaptive moving mesh schemes for other nonlinear wave equations in the WKI class.

More details about exact solutions, fully discretizations, and numerical computations of self-adaptive moving mesh schemes for the coupled SP equation, the complex SP equation, and their generalized equations will be discussed in our forthcoming papers.

\section{Appendix}

The multi-soliton solutions of the coupled SP equation (57) and (58) are given by the following formula:

$$
\begin{aligned}
& u=\frac{g}{f}, \quad v=\frac{h}{f}, \quad \rho=1-2(\ln f)_{X T}, \\
& x=X_{0}+\int_{X_{0}}^{X} \rho(\tilde{X}, T) d \tilde{X}, \quad t=T, \\
& f=\left|\begin{array}{cc}
\mathcal{A}_{N} & I_{N} \\
-I_{N} & \mathcal{B}_{N}
\end{array}\right|=\left|I_{N}+\mathcal{A}_{N} \mathcal{B}_{N}\right|, \\
& g=\left|\begin{array}{ccc}
\mathcal{A}_{N} & I_{N} & \mathbf{e}_{N}^{(1)^{\top}} \\
-I_{N} & \mathcal{B}_{N} & \mathbf{0}^{\top} \\
\mathbf{0} & -\mathbf{a}_{N}^{(1)} & 0
\end{array}\right|, \\
& h=\left|\begin{array}{ccc}
\mathcal{A}_{N} & I_{N} & \mathbf{0}^{\top} \\
-I_{N} & \mathcal{B}_{N} & \mathbf{a}_{N}^{(2)} \\
\mathbf{e}_{N}^{(2)} & \mathbf{0} & 0
\end{array}\right|,
\end{aligned}
$$

where

$$
\begin{gathered}
\mathcal{A}_{N}=\left(\begin{array}{cccc}
\frac{\mathrm{e}^{\xi_{1}+\xi_{N+1}}}{4\left(\frac{1}{p_{1}}+\frac{1}{p_{N+1}}\right)} & \frac{\mathrm{e}^{\xi_{1}+\xi_{N+2}}}{4\left(\frac{1}{p_{1}}+\frac{1}{p_{N+2}}\right)} & \cdots & \frac{\mathrm{e}^{\xi_{1}+\xi_{2 N}}}{4\left(\frac{1}{p_{1}}+\frac{1}{p_{2 N}}\right)} \\
\frac{\mathrm{e}^{\xi_{2}+\xi_{N+1}}}{4\left(\frac{1}{p_{2}}+\frac{1}{p_{N+1}}\right)} & \frac{\mathrm{e}^{\xi_{2}}+\xi_{N+2}}{4\left(\frac{1}{p_{2}}+\frac{1}{p_{N+2}}\right)} & \cdots & \frac{\mathrm{e}^{\xi_{2}}+\xi_{2 N}}{4\left(\frac{1}{p_{2}}+\frac{1}{p_{2 N}}\right)} \\
\vdots & \vdots & \ddots & \vdots \\
\frac{\mathrm{e}^{\xi_{N}+\xi_{N+1}}}{4\left(\frac{1}{p_{N}}+\frac{1}{p_{N+1}}\right)} & \frac{\mathrm{e}^{\xi_{N}+\xi_{N+2}}}{4\left(\frac{1}{p_{N}}+\frac{1}{p_{N+2}}\right)} & \cdots & \frac{\mathrm{e}^{\xi_{N}+\xi_{2 N}}}{4\left(\frac{1}{p_{N}}+\frac{1}{p_{2 N}}\right)}
\end{array}\right), \\
\mathcal{B}_{N}=\left(\begin{array}{cccc}
\frac{a_{1} a_{N+1}}{1 / p_{1}+1 / p_{N+1}} & \frac{a_{2} a_{N+1}}{1 / p_{2}+1 / p_{N+1}} & \cdots & \frac{a_{N} a_{N+1}}{1 / p_{N}+1 / p_{N+1}} \\
\frac{a_{1} a_{N+2}}{1 / p_{1}+1 / p_{N+2}} & \frac{a_{2} a_{N+2}}{1 / p_{2}+1 / p_{N+2}} & \cdots & \frac{a_{N} a_{N+2}}{1 / p_{N}+1 / p_{N+2}} \\
\vdots & \vdots & \ddots & \vdots \\
\frac{a_{1} a_{2 N}}{1 / p_{1}+1 / p_{2 N}} & \frac{a_{2} a_{2 N}}{1 / p_{2}+1 / p_{2 N}} & \cdots & \frac{a_{N} a_{2 N}}{1 / p_{N}+1 / p_{2 N}}
\end{array}\right),
\end{gathered}
$$

and $I_{N}$ is the $N \times N$ identity matrix, $\mathbf{a}^{\top}$ is the transpose of $\mathbf{a}$,

$$
\begin{aligned}
& \mathbf{a}_{N}^{(1)}=\left(a_{1}, a_{2}, \cdots, a_{N}\right), \quad \mathbf{e}_{N}^{(1)}=\left(e^{\xi_{1}}, e^{\xi_{2}}, \cdots, e^{\xi_{N}}\right) \\
& \mathbf{a}_{N}^{(2)}=\left(a_{N+1}, a_{N+2}, \cdots, a_{2 N}\right) \\
& \mathbf{e}_{N}^{(2)}=\left(e^{\xi_{N+1}}, e^{\xi_{N+2}}, \cdots, e^{\xi_{2 N}}\right) \\
& \mathbf{0}=(0,0, \cdots, 0), \\
& \xi_{i}=p_{i} X+\frac{1}{p_{i}} T, \quad 1 \leq i \leq 2 N
\end{aligned}
$$

\section{Author details}

${ }^{1}$ Department of Mathematics, The University of Texas-Pan American, Edinburg, Texas 78541, USA. ${ }^{2}$ Department of Applied Mathematics, School of Fundamental Science and Engineering, Waseda University, 3-4-1 Okubo, Shinjuku-ku, Tokyo 169-8555, Japan. ${ }^{3}$ Department of Mathematics, Kobe University, Rokko, Kobe 657-8501, Japan.

Received: 10 March 2014 Accepted: 14 March 2014 Published online: 12 September 2014 


\section{References}

1. Ablowitz, M.J., Ladik, J.F.: Nonlinear differential-difference equations. J. Math. Phys. 16, 598-603 (1975)

2. Ablowitz, M.J., Ladik, J.F.: Nonlinear differential-difference equations and Fourier analysis. J. Math. Phys. 17, 1011-1018 (1976)

3. Ablowitz, M.J., Ladik, J.F.: A nonlinear difference scheme and inverse scattering. Stud. Appl. Math. 55, 213-229 (1977)

4. Ablowitz, M.J., Ladik, J.F.: On the solution of a class of nonlinear partial difference equations. Stud. Appl. Math. 57, 1-12 (1977)

5. Ablowitz, M.J., Segur, H.: Solitons and Inverse Scattering Transform SIAM, Philadelphia (1981)

6. Bobenko, A.I., Suris, Y.B.: Discrete Differential Geometry, Graduate Studies in Mathematics, Vol. 98. AMS, Rhode Island (2008)

7. Bobenko, A.I., Seiler, R. (eds.): Discrete Integrable Geometry and Physics, Oxford Lecture Series in Mathematics and Its Applications, Vol. 16. Oxford Univ. Press, Oxford (1999)

8. Chung, Y., Jones, C.K.R.T., Schäfer, T., Wayne, C.E.: Ultra-short pulses in linear and nonlinear media. Nonlinearity. 18, 1351-1374 (2005)

9. Dimakis, A., Müller-Hoissen, F.: Bidifferential calculus approach to AKNS hierarchies and their solutions. SIGMA. 6, 055 (2010)

10. Feng, B.F.: Complex short pulse and coupled complex short pulse equations. arXiv:1312.6431 (2013)

11. Feng, B.F., Inoguchi, J., Kajiwara, K., Maruno, K., Ohta, Y.: Discrete integrable systems and hodograph transformations arising from motions of discrete plane curves. J. Phys. A. 44, 395201 (2011)

12. Feng, B.F., Inoguchi, J., Kajiwara, K., Maruno, K., Ohta, Y.: Integrable discretizations of the Dym equation. Front. Math. China. 8, 1017-1029 (2013)

13. Feng, B.F., Maruno, K., Ohta, Y.: A self-adaptive moving mesh method for the Camassa-Holm equation. J. Comput. Appl. Math. 235, 229-243 (2010)

14. Feng, B.F., Maruno, K., Ohta, Y.: Integrable discretizations of the short pulse equation. J. Phys. A. 43, 085203 (2010)

15. Grammaticos, B., Kosmann-Schwarzbach, Y., Tamizhmani, T. (eds.): Discrete Integrable Systems, Lecture Notes in Physics, Vol. 644 Springer-Verlag, Berlin (2004)

16. Hirota, R.: Nonlinear partial difference equations. I. A difference analogue of the Korteweg-de Vries equation. J. Phys. Soc. Jpn. 43, 4116-4124 (1977)

17. Hirota, R.: Nonlinear partial difference equations. II. Discrete-time Toda equation. J. Phys. Soc. Jpn. 43, 2074-2078 (1977)

18. Hirota, R.: Nonlinear partial difference equations. III. Discrete sine-Gordon equation. J. Phys. Soc. Jpn. 43, 2079-2086 (1977)

19. Hirota, R.: Nonlinear partial difference equations. IV. Bäcklund transformation for the discrete-time Toda equation. J. Phys. Soc. Jpn. 45, 321-332 (1978)

20. Hirota, R.: Nonlinear partial difference equations. V. Nonlinear equations reducible to linear equations. J. Phys. Soc. Jpn. 46, 312-319 (1979)

21. Hirota, R., Tsujimoto, S.: Note on "New coupled integrable dispersionless equations". J. Phys. Soc. Jpn. 63, 3533 (1994)

22. Ishimori, Y.: A relationship between the Ablowitz-Kaup-Newell-Segur and Wadati-Konno-Ichikawa schemes of the inverse scattering method. J. Phys. Soc. Jpn. 51, 3036-3041 (1982)

23. Ishimori, Y.: On the modified Korteweg-de Vries soliton and the loop soliton. J. Phys. Soc. Jpn. 50, 2471-2472 (1981)

24. Kakuhata, H., Konno, K.: Interaction among growing, decaying and stationary solitons for coupled integrable dispersionless equations. J. Phys. Soc. Jpn. 64, 2707-2709 (1995)

25. Kakuhata, H., Konno, K.: Novel solitonic evolutions in a coupled integrable, dispersionless system. J. Phys. Soc. Jpn. 65, 340-341 (1996)

26. Konno, K.: Integrable coupled dispersionless equations. Applicable Anal. 57, 209-220 (1995)

27. Konno, K., Ichikawa, Y., Wadati, M.: A loop soliton propagating along a stretched rope. J. Phys. Soc. Jpn. 50, 1025-1026 (1981)

28. Konno, K., Oono, H.: New coupled integrable dispersionless equations. J. Phys. Soc. Jpn. 63, 377-378 (1994)

29. Konno, K., Oono, H.: Reply to note on "New coupled integrable dispersionless equations". J. Phys. Soc. Jpn. 63, 3534 (1994)

30. Kotlyarov, $\mathrm{V}$ :: On equations gauge equivalent to the sine-Gordon and Pohlmeyer-Lund-Regge equations. J. Phys. Soc. Jpn. 63, 3535-3537 (1994)

31. Matsuno, Y.: A novel multi-component generalization of the short pulse equation and its multisoliton solutions. J. Math. Phys. 52, 123702 (2011)
32. Matsuno, Y.: Multiloop soliton and multibreather solutions of the short pulse model equation. J. Phys. Soc. Jpn. 76, 084003 (2007)

33. Matsuno, Y.: Soliton and periodic solutions of the short pulse model equation. In: Lang, SP, Bedore, H (eds.) Handbook of Solitons: Research, Technology and Applications, pp. 541-586. Nova Publishers, Hauppauge, New York, (2009)

34. Ohta, Y., Maruno, K., Feng, B.F.: An integrable semi-discretization of the Camassa-Holm equation and its determinant solution. J. Phys. A. 41, 355205 (2008)

35. Ohta, Y., Maruno, K., Feng, B.F.: Integrable discretizations for the short-wave model of the Camassa-Holm equation. J. Phys. A. 43, 265202 (2010)

36. Rogers, S., Schief, W.K.: Bäcklund and Darboux Transformations: Geometry and Modern Applications in Soliton Theory. Cambridge University Press, Cambridge (2002)

37. Sakovich, A., Sakovich, S.: Solitary wave solutions of the short pulse equation. J. Phys. A. 39, L361-L367 (2006)

38. Sakovich, A., Sakovich, S.: The short pulse equation is integrable. J. Phys. Soc. Jpn. 74, 239-241 (2005)

39. Schäfer, T., Wayne, C.E.: Propagation of ultra-short optical pulses in cubic nonlinear media. Physica D. 196, 90-105 (2004)

40. Suris, Y.B.: The Problem of Integrable Discretization: Hamiltonian Approach. Basel, Birkhäuser (2003)

41. Vinet, L., Yu, G.-F.: Discrete analogues of the generalized coupled integrable dispersionless equations. J. Phys. A. 46, 175205 (2013)

42. Vinet, L., Yu, G.-F.: On the discretization of the coupled integrable dispersionless equations. J. Nonlinear Math. Phys. 20, 106-125 (2013)

43. Wadati, M., Konno, K., Ichikawa, Y.: New integrable nonlinear evolution equations. J. Phys. Soc. Jpn. 47, 1698-1700 (1979)

44. Wadati, S., Sogo, K.: Gauge transformations in soliton theory. J. Phys. Soc. Jpn. 52, 394-398 (1983)

doi:10.1186/s40736-014-0008-7

Cite this article as: Feng et al:: Self-adaptive moving mesh schemes for short pulse type equations and their Lax pairs. Pacific Journal of Mathematics for Industry 2014 6:8.

\section{Submit your manuscript to a SpringerOpen ${ }^{\circ}$ journal and benefit from:}

- Convenient online submission

- Rigorous peer review

- Immediate publication on acceptance

- Open access: articles freely available online

- High visibility within the field

- Retaining the copyright to your article

Submit your next manuscript at $>$ springeropen.com 